\title{
Measuring adhesive bond strength and microhardness of multilayer composite wear-resistant coating
}

\author{
Michael M. Stebulyanin, Alina A. Gurkina a , Alexander A. Shein And \\ Natalia Yu. Cherkasova \\ Moscow State University of Technology "STANKIN", 1 Vadkovsky per., Moscow GSP-4, 127994, Russian Federation
}

Received 9 November 2016, Accepted 28 November 2016

\begin{abstract}
Presently, the modifying multilayer composite coatings have been increasingly used for upgrading performance characteristics of different products. The quality of these coatings and, consequently, efficiency of product properties modification strongly depends on the coating properties such as hardness and adhesive strength of the "coating-substrate" interface and coating interlayer boundaries. Therefore, in present paper a problem of improving accuracy of measurement and control of desired parameters of multilayer composite wear-resistant coatings is considered. An analysis of applied control methods and their quality indicators and capabilities is carried out. An approach to control the surface characteristics of products having the coatings as well as results of research of adhesion parameters and hardness of a wide range of the latest-generation multilayer composite wear-resistant coatings are presented.
\end{abstract}

Key words: Multilayer composite wear-resistant coatings / adhesion / hardness / scribing / scratch test

\section{Introduction}

In manufacturing products with modifying coatings (for example, cutting tools, friction pairs, medical products), the measurement and control of the main coating properties like adhesive bond strength, microhardness, thickness, chemical and phase composition, surface roughness, friction factor etc. are the most important tasks of the processes used for deposition of multilayer composite coatings with desired properties and their reliability and longevity control.

For a variety of specific features of coatings as objects of control, a special approach for measuring their parameters is required. The coating is a thin film with thickness of from $10 \mathrm{~nm}$ to $10 \mu \mathrm{m}$, with high hardness of up to $9000 \mathrm{HV}$ (for diamond-like coatings) being deposed to the substrate with significantly lower hardness and greater thickness. In recent years, the last generation of multilayer composite coatings with nanometricgrain structure and sub layer thickness have been developed and widely introduced in production $[1,2]$. The control of the parameters of those coatings may be difficult because of the sublayers having variable hardness and the interlayer adhesion. It should be noticed that the adhesive bond strength on the "substrate-coating" interface boundaries and the interlayer adhesion as well as the microhardness

\footnotetext{
${ }^{\mathrm{a}}$ Corresponding author: a.gurkina@stankin.ru
}

of multilayer composite coatings are the most important and highly-controlled parameters strongly influencing on the coatings longevity and performance parameters of the coated products.

In present research, in dexable inserts made of tungsten carbide VK8 (92\% WC and 8\% Co) with a range of multilayer composite coatings produced with the use of innovative vacuum arc deposition with ion flux filtration [3] and inserts with a standard single-layer TiN coating were used as test objects. To compare the results of the hardness measurement, inserts made of high-speed steel R6M5 $(6 \% \mathrm{~W}, 5 \% \mathrm{Mo}$ and $4 \% \mathrm{Cr})$ with the same coatings were also used. The research objective is the choice of optimum approaches to the control of adhesive bond strength and the microhardness measurement of multilayer composite wear-resistant coatings allowing highest accuracy and consistent results.

\section{Discussion}

\subsection{Adhesion.Qualitative evaluation}

For the consistent express evaluation of the strength of adhesive bond between coating and substrate, the test approach based on VDI-3198 standard [4] is used. According to this approach, the evaluation of the adhesive bond strength in relation to the substrate is performed by 
the cracks around the indenter imprint (DIN 50103 T1 3.84 ) and the coating delamination with consecutive assignment the adhesion strength class index. For this purpose, the acceptable strength class indexes are HF $1-\mathrm{HF}$ 4, while the HF 5 - HF 6 are unallowable. The apparent advantage of this method is its simplicity and availability. However, there are some obvious disadvantages in the VDI-3198 test approach [5]. In particular, for relatively soft coatings, the delamination from the substrate may take place without crack formation or fracture. The large imprint size $(0.3-0.5 \mathrm{~mm})$ does not allow using this approach to control the adhesive bond strength directly on the working surfaces of the samples. It is also noticeable that this method is not suitable for the multilayer coatings where the adhesive bond between single layers and sublayers is also present.

When the Vickers hardness machine is used instead of the Rockwell machine, the equivalent result can be obtained, however the indenter imprint size will be much smaller, and it is possible to estimate the adhesive bond between the coating layers and sublayers (Fig. 1a).

The objective estimation of the adhesive bond strength may be performed by the spherical grinding using the CALOWEAR CAW-S-AE-000 coating-thickness gauge (Fig. 1b). In that case, the conditions modelling the real cutting process arise. It is possible to estimate not only the adhesion between coating and substrate, but also the adhesion interaction between single coating layers.

\section{Adhesion. Quantitative evaluation}

The quantitative evaluation approaches are much more meaningful and allow not only evaluating the adhesive interaction quality but also obtaining the numeric data. The main methodology used to evaluate and measure the adhesive bond strength of the multilayer composite coating is the scratch test. The adhesive bond strength evaluation approach basing on the scratch test is used mainly for the flat surfaces with coatings, while performing research for the complex-formed samples using this method is practically impossible.

In this research, the Nanovea scratch tester was applied. The conical diamond indenter with the tip angle of $120^{\circ}$ and the tip radius of $100 \mu \mathrm{m}$ is used. The load increased linearly from $0.05 \mathrm{~N}$ to the final value of $40 \mathrm{~N}$. The scratches length is $5 \mathrm{~mm}$. Delamination of local coating volumes along the scratch can be detected directly during the test, e.g. by estimation of the acoustic emission signal parameters using the piezoelectric sensor. The cracks and delamination of the coating can be registered by the out coming acoustic emission signal with the maximum amplitude. For the statistical confidence of the experimental study, each test was repeated three times.

Using the obtained graphs, two parameters were detected $[6]$ :

1. First critical load $L_{\mathrm{C} 1}$ causing the initial cracks along the scratch;
Table 1. Adhesive bond strength of multilayer coatings on the tungsten carbide substrate.

\begin{tabular}{ccc}
\hline Coating structure & $L_{C 1}$ & $L_{C 2}$ \\
\hline TiN & 20 & 25 \\
Ti-TiN-(TiCrAl)N & 28 & 36 \\
Zr-ZrN-(ZrCrAl)N & 36 & 39 \\
Zr-ZrN-(ZrNbCrAl)N & 34 & 39 \\
Cr-CrN-(TiCrAl)N & 21 & $>40$ \\
Ti-TiN-(NbZrTi)N & 26 & 33 \\
Ti-TiN-(NbZrAl)N & 25 & 31 \\
Ti-TiN-(NbZrTiAl)N & 34 & $>40$ \\
Cr-CrAlN-(NbZrCrAl)N ${ }^{*}$ & - & 28 \\
\hline
\end{tabular}

* The initial coating damage sites are nearly non-existent, thus only $L_{\mathrm{C} 2}$ parameter is detected.

2. Second critical (crushing) load $L_{\mathrm{C} 2}$ causing complete destruction (delamination) of the coating along the scratch.

Analyzing the acoustic emission signal allows evaluation of the critical (crushing) load $L_{\mathrm{C} 2}$. With increase of the normal load applied to the diamond indenter, the measured acoustic signal value grows too.

During the experiment test, the friction force was also registered. Its behavior is identical to the acoustic emission signal change, i.e. it increases with the increase of the load applied.

In Figure 2a, the sample with the scratch made with the maximum load to the indenter (final segment) is shown. This image allows to observe the fragments of the multilayer Ti-TiN-(TiCrAl)N with complete delamination from the tungsten carbide substrate that is corresponding to the final stage of destruction called wedging spallation. The delamination of single layers is also observed. This wedging spallation is featured to multilayer coatings. As a comparison, the final segment of the scratch on the monolayer TiN coating looking like an uniform groove without elements of wedging spallation is shown in Figure 2b.

The parameters of acoustic emission signal when evaluating the adhesive bond strength between tungsten carbide substrate and multilayer Ti-TiN-( TiCrAl)N coating noticeably differ from ones for the monolayer TiN coating. The acoustic emission signal for the multilayer TiTiN-(TiCrAl)N coating is more complex and have more peaks caused not only by the delamination of the coating as a whole from the substrate, but also by splitting into sub layers during the test in Figure 3.

The measurement results of the adhesive bond strength for the monolayer TiN coating and for several multilayer coatings deposed to the tungsten carbide substrate (VK8) are presented in Table 1.

For a quantitative evaluation and measurement of the adhesive bond strength of the multilayer composite coating, a pendulum scratch test is applied [7]. In this test, the indenter tip moves along the defined trajectory (circular arc) leaving a scratch on sampling material and going deeper to the $h_{\max }$ depth with a subsequent retract. 


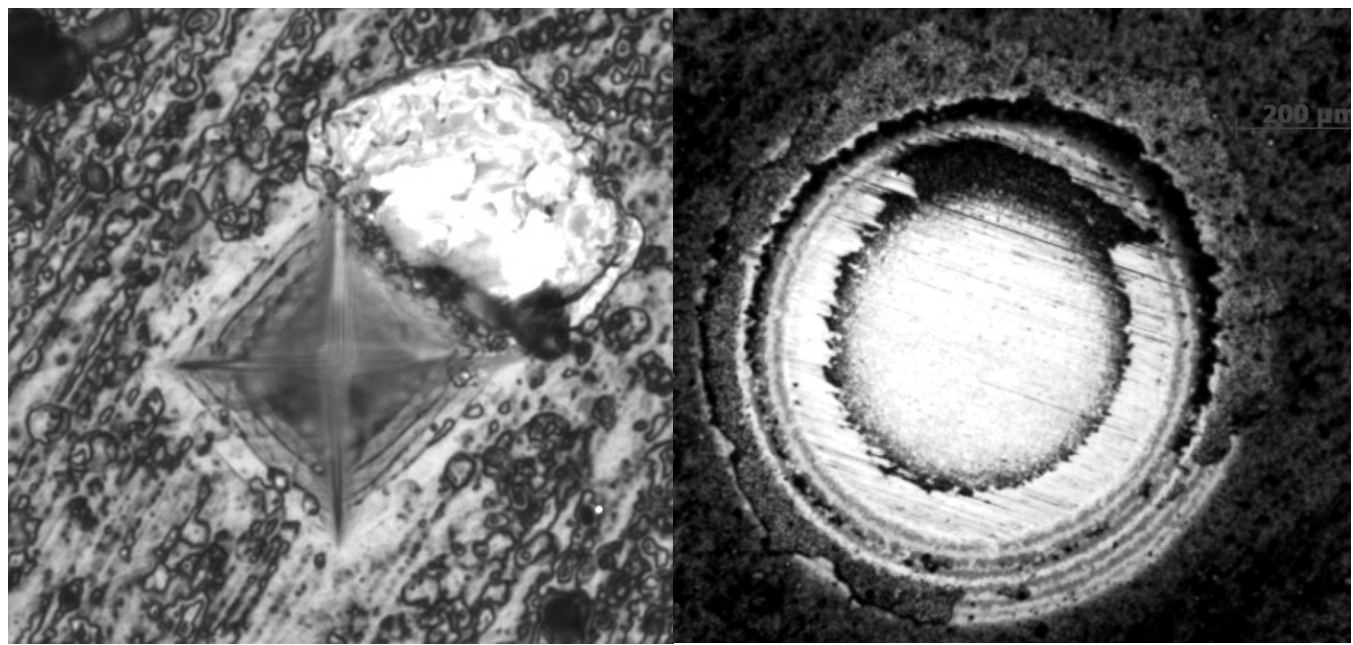

Fig. 1. Low adhesion between coating and substrate and interlayer adhesion detected with: (a) Vickers pyramid hardness machine and (b) using CALOWEAR CAW-S-AE-000 coating-thickness gauge after the spherical grinding of the sample.

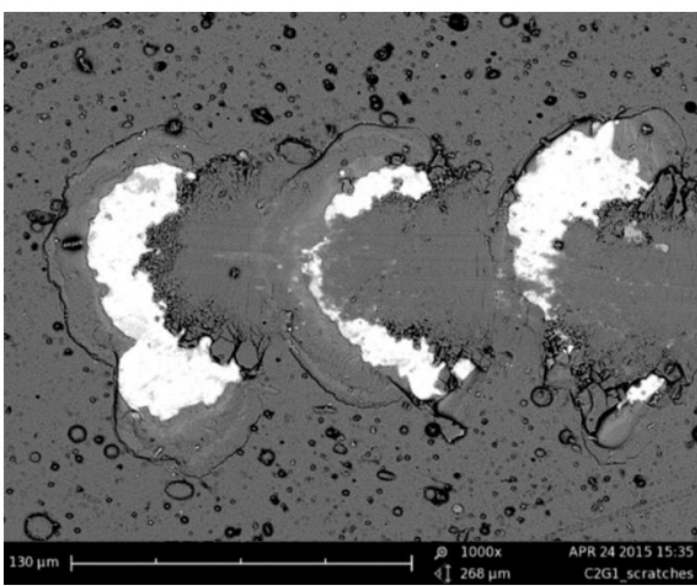

(a)

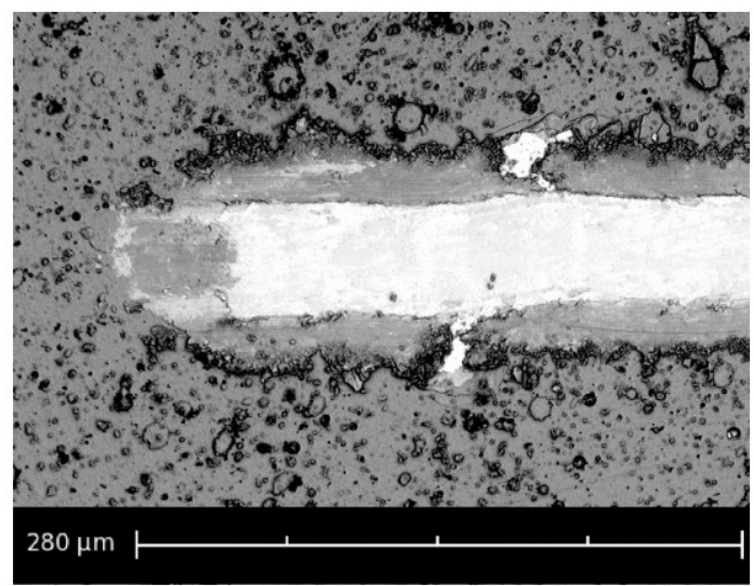

(b)

Fig. 2. The final part of the scratch produced by scribing of: (a) multilayer Ti-TiN-(TiCrAl)N and (b) single-layer TiN coatings.

Among other approaches used for the adhesive bond strength definition, the laser shock adhesion test (LASAT) should be mentioned. This method involves measuring size of interphase cracks arising as a result of laser shocks with laser beam power density multiplication [8]. In addition, this approach allows both qualitative and quantitative (by means of changing the delamination area depending on the laser beam power density) evaluation of the adhesive bond strength. However, this method should be additionally elaborated because it is a quite new and presently there is insufficient data amount.

\section{Coating hardness measurement}

Measuring coating hardness using traditional methods (e.g., with Vickers or Rockwell hardness tests) has some difficulties caused by the architecture and properties of the multilayer composite coatings. In particular, under a standard indenting load $(5-50 \mathrm{~g})$ the indentation depth is much larger than the coating depth. This means that obtained microhardness value is not the coating microhardness but certain integral hardness of substrate and coating. There are several approaches to solve this task. The first approach deals with the "substrate-coating" system as a two-phase statistical system where one phase is coating and the second one is substrate. Thus, preliminary definition of the coating thickness allows singling out the coating hardness as one component of the integral hardness of the "substrate-coating" system.

According to this approach, the following study was carried out. At first, the coating depth equal to $3 \pm 0.2 \mu \mathrm{m}$ is defined by the spherical grinding. Next, the hardness of the substrate without coating was measured. For this purpose, the sample was placed to the standard Vickers microhardness machine with the quadrangular pyramidindenter under applied indenting load $F$ which value is enough to produce an imprint with a diagonal of about $4 \mu \mathrm{m}$. After that, the coating hardness itself was defined on the following ground. The properties of the statisti- 

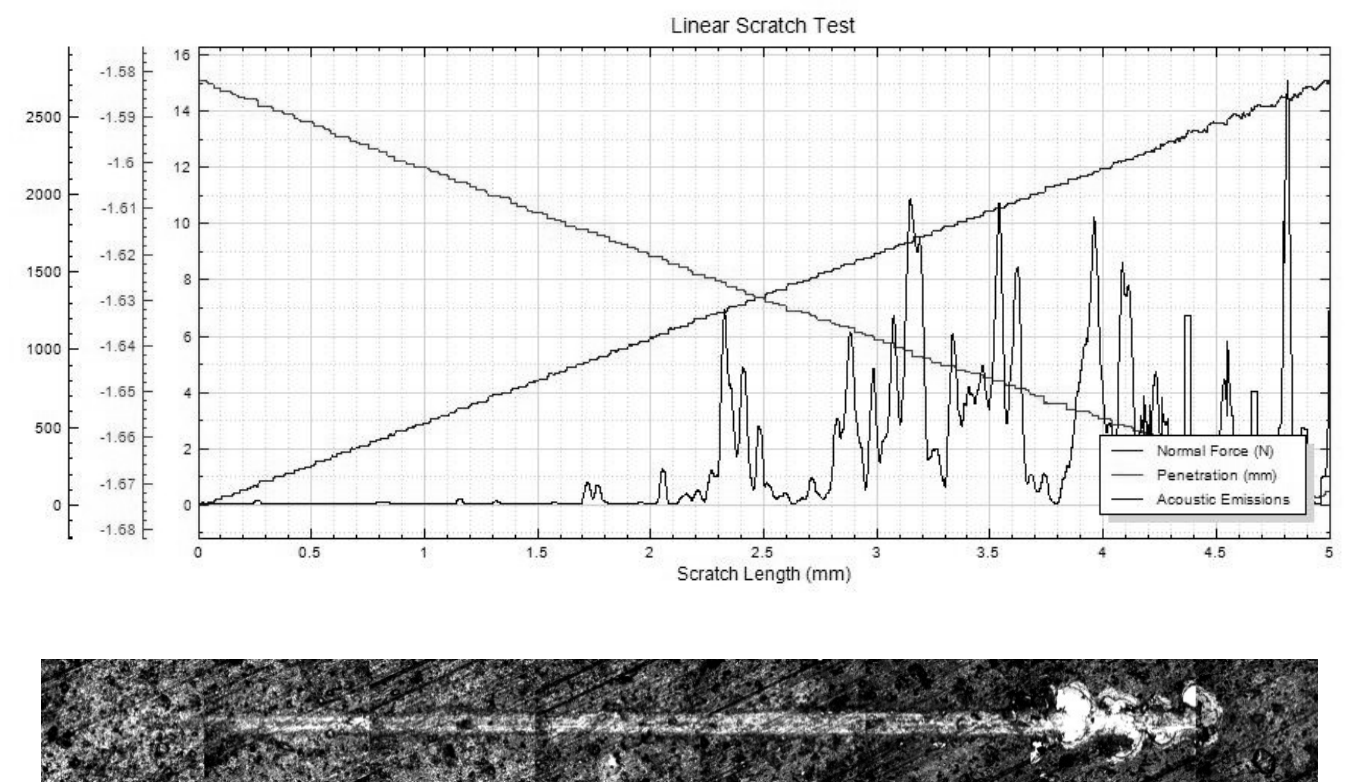

Fig. 3. Graph of variance of the load and signal obtained from the acoustic emission sensor (top) and panorama of the scratch on the sample with the multilayer $\mathrm{Ti}-\mathrm{TiN}-(\mathrm{NbZrAl}) \mathrm{N}$ coating (bottom).

cal system where one phase is coating and another one is substrate conform to the rule of additivity, consequently:

$$
H V_{\text {syst }}=n H V_{\text {coat }}+(1--n) H V_{\text {sub }},
$$

where $H V_{\text {syst }}, H V_{\text {coat }}$ and $H V_{\text {sub }}$ are hardness of system, coating and substrate, respectively; $n$ is coating hardness amount in the total system hardness. This amount is equal to the ratio of area of imprint on the coating to the total area of imprint in the system.

From (1) we obtain:

$$
H V_{\text {coat }}=\frac{H V_{\text {syst }}-(1-n) H V_{\text {sub }}}{n}
$$

For thequadrangular pyramidindenter with the tip angle of $136^{\circ}$ :

$$
n=1-\frac{\left(h-h_{\text {coat }}\right)^{2}}{h^{2}}
$$

where $\mathrm{h}_{\text {coat }}$ is coating thickness; $\mathrm{h}$ is total indenting depth.

Geometrically,

$$
n=1-\frac{\left(0,14 d-h_{\text {coat }}\right)^{2}}{0,0196 d^{2}}
$$

where $d$ is the imprint diagonal.

The microhardness was measured using the Instron Wilson Hardness Group Tukon 2500 machine under the load of $1 \mathrm{~kg}$. Totally five hardness tests were performed with each sample.

It should be noted that this method did not give adequate results for the multilayer coatings on the tungsten carbide and high-speed steel substrates. For instance, the hardness of the Ti-TiN-(NbZrTi)N coating on the highspeed steel R6M5 according to the obtained results is
$1040 H V_{1,0}$ while the hardness of the same coating on the tungsten carbide VK8 is $1956 H V_{1,0}$. Further, the hardness of the Ti-TiN-(NbZrTi)N coating carried out with the nanoindentation method is $34 \mathrm{GPa}$ both for tungsten carbide and high-speed steel substrates. Thus, it is expected that the contribution of the substrate hardness to the total hardness of the system when measuring with pressing through the coating cannot be evaluated only geometrically.

Another method for the coating hardness measurement is a low force indentation (less than $0.01 \mathrm{~N}$ ). In that case, a standard Vickers hardness machine with a load of $5-10 \mathrm{~g}$ is used. Indenter intrudes into the coating without pressing through it and not affecting the substrate structure. Main disadvantage of this method is a poor imprint clarity making difficult its accurate measurement. The measurement error is up to $20 \%$ does not allow considering this method as an accurate and adequate one. Besides, only a few Vickers machines can work well under such low loads.

The most accurate method for coating hardness measurement including multilayer ones is nanoindentation.

Measuring hardness of a wide range of multilayer coatings were performed with the Nano Test machine that measures a vertical movement of diamond indenter during its contact with the studied surface. The force applied to the indenter constantly grows. The force magnitude and its action time are recorded. Basing on the obtained results analysis, a retraction curve considering plasticity and elasticity of material is constructed. The Berkovich in denterisused. The load range is $0-20 \mathrm{mN}$, indentation depth is $0-200 \mathrm{~nm}$. The obtained data are presented in Table 2.

Comparing hardness of coatings on the substrates made of tungsten carbide (VK8) and high-speed steel 
Table 2. Hardness of coatings deposed to the tungsten carbide substrate.

\begin{tabular}{ccc}
\hline No. & Coating structure & Hardness HV, GPa \\
\hline 1 & Zr-ZrN-(ZrCrAl)N & 38 \\
2 & Ti-TiN-(NbZr-Al)N & 39 \\
3 & Zr-ZrN-(ZrNbCrAl)N & 42 \\
4 & Ti-TiN-(TiCrAl)N & 40 \\
5 & Cr-CrN-(TiCrAl)N & 41 \\
6 & Ti-TiN-(NbZrTiAl)N & 36 \\
7 & Cr-CrAlN-(NbZrCrAl)N & 38 \\
8 & Ti-TiN-(NbZrTi)N & 34 \\
9 & TiN & 30 \\
10 & Substrate with no coating & 28 \\
\hline
\end{tabular}

(R6M5)deposed under similar conditions does not show substantial difference (hardness value deviation is less than $5 \%$ ). This result confirms that the nanoindentation method provides the measurement of coating hardness itself without influence of the substrate hardness.

It is important to note that all the studies were conducted at room temperature while in cutting process the cutter operates at temperatures of $700{ }^{\circ} \mathrm{C}$ and higher. At these temperatures, the change of chemical and physical properties of materials occurs, including hardness and adhesion bond. Some researchers (in particular [9]) justify the need to control the main coating parameters at the temperatures close to the cutting temperature. To conduct corresponding studies special equipment is required, whereas even large research centers rarely can afford it. However, regarding the temperature is necessary and can be performed using special approaches.

\section{Summary}

Adhesion and hardness are most frequently controlled properties of wear-resistant coatings including the multilayer ones. There is a number of approaches for the qualitative or quantitative control of adhesion bond strength as well as different methods of the coatings hardness measurement. To control the coating adhesion bond strength with regard to the substrate, the traditional scribing of the diamond indenter with recording of the acoustic emission signal for the "substrate-coating" system is used. In addition, the methods of spherical grinding and laser shock adhesion can be applied. When estimating the adhesive properties of the multilayer coatings, it is important to take into consideration the interlayer adhesion which can be estimated either by spherical grinding (qualitative analysis) or scribing (quantitative analysis).

The obtained results afford the conclusion that the most accurate and valid method to estimate the microhardness of the "coating-substrate" system is the nanoindentation with the load not exceeding $20 \mathrm{mN}$.
The "geometrical" approach considering the "substrate-coating" system as a two-phase statistical system does not provide a fair accuracy, and neither the microhardness measurement using a standard Vickers machine with the load not exceeding 5-10 g.

Acknowledgements. The work is performed with financial support of the Russian Science Foundation within the Agreement No. 14-29-00297 of August 6, 2014.

\section{References}

[1] S.N. Grigoriev, A.A. Vereschaka, A.S. Vereschaka, A.A. Kutin, Cutting tools made of layered composite ceramics with nano-scale multilayered coatings, Procedia CIRP 1 (2012) 318-323

[2] A.S. Vereschaka, S.N. Grigoriev, E.S. Sotova, A.A. Vereschaka, Improving the efficiency of the cutting tools made of mixed ceramics by applying modifying nanoscale multilayered coatings, Adv. Mater. Res. 712-715 (2013) 391-394

[3] A.A. Vereshchaka, A.S. Vereshchaka, O. Mgaloblishvili, M.N. Morgan, A.D. Batako, Nano-scale multilayeredcomposite coatings for the cutting tools, Int. Adv. Manuf. Technol. 72 (2014) 303-317

[4] T. Cselle, Nanostracturierte Schichten in der Werkstaff, Platit AG. Warkzeugtagung, 2002

[5] N. Vidakis, A. Antoniadis, N. Bilalis, The VDI-3198 indentation test evaluation of a reliable qualitative control for layered compounds, J. Mater. Process. Technol. 143144 (2003) 481-485

[6] ASTM C1624-05 Standard Test Method for Adhesion Strength and Mechanical Failure Modes, 2010

[7] B.Y. Mokritskii, D.A. Pustovalov, A.A. Vereschaka, Comparative evaluation of efficiency of metal-cutting tool by method of pendulum scribing, Appl. Mech. Mater. 798 (2015) 452-456

[8] G. Bégué, G. Fabre, V. Guipont, M. Jeandin, P. Bilhe, J.Y. Guédou, F. Lepoutre, Laser Shock Adhesion Test (LASAT) of EB-PVD TBCs: Towards an industrial application, Surf. Coat. Technol. 237 (2013) 305-312

[9] B.D. Beake, J.F. Smith, A. Gray, G.S. Fox-Rabinovich, S.C. Veldhuis, J.L. Endrino, Investigating the correlation between nano-impact fracture resistance and hardness/modulus ratio from nanoindentation at $25-500{ }^{\circ} \mathrm{C}$ and the fracture resistance and lifetime of cutting tools with Til-xAlxN ( $\mathrm{x}=0.5$ and 0.67$)$ PVD coatings in milling operations, Surf. Coat. Technol. 201 (2007) 45854593 